\title{
Immediate Total Elbow Arthroplasty Following Olecranon Osteotomy: A Case Report
}

\author{
Lee M. Reichel ${ }^{*}$, Cody D. Hillin, Charles A. Reitman
}

Department of Orthopedic Surgery, Baylor College of Medicine, Ben Taub General Hospital, Houston, USA.

Email: *leereichel@gmail.com

Received May $6^{\text {th }}, 2013$; revised June $8^{\text {th }}, 2013$; accepted June $22^{\text {nd }}, 2013$

Copyright (C) 2013 Lee M. Reichel et al. This is an open access article distributed under the Creative Commons Attribution License, which permits unrestricted use, distribution, and reproduction in any medium, provided the original work is properly cited.

\begin{abstract}
Consistent with an aging population, the overall number of distal humeral fractures in the elderly is increasing. Indications for application of acute total elbow arthroplasty (TEA) in the setting of distal humeral fractures are still being defined. A variety of factors including chronologic age, physiologic age, bone quality, presence of pre-existing arthritis and pre-existing medical conditions need to be considered. Optimally the decision to proceed with TEA verses open reduction internal fixation (ORIF) is made preoperatively. The need to abandon ORIF may be not be apparent until after fracture exposure, and the presence of an olecranon osteotomy makes performing TEA challenging. A case is presented of acute conversion from ORIF to TEA following olecranon osteotomy, utilizing internal fixation bridging the ulnar component and its cement mantle.
\end{abstract}

Keywords: Distal Humerus Fracture; Elderly; Total Elbow Arthroplasty; Olecranon Osteotomy

\section{Historical Perspective}

In younger patients, a generally accepted set of principles have evolved for distal humeral fracture management, but best treatment practices in the elderly have not been clearly [1]. Recent debate has focused on whether elderly patients benefit more from open reduction internal fixation verses TEA for displaced intra-articular fractures. Treatment strategies for elderly patients are important as the absolute number of distal humeral fractures in patients greater than 60 years old is increasing secondary to population dynamics [2,3]. Mckee et al. performed a randomized, prospective trial comparing outcomes of ORIF verses TEA in the elderly and found that at two years patients who underwent TEA had more predictable and better functional outcomes than the ORIF group and thus supported TEA as a preferred alternative for fractures not amenable to stable internal fixation [4].

It is generally accepted that olecranon osteotomy is contra-indicated when performing TEA in order to maintain competency of the elbow extensor mechanism. Other authors have alluded to conversion of attempted ORIF to TEA, but have not given specifics on how this was done, or if this was done following an olecranon osteotomy $[4,5]$. Olecranon osteotomy allows maximal visualization

\footnotetext{
"Corresponding author.
}

of the distal humerus, but is clearly a disadvantage if a conversion to TEA is needed. Marra et al. reviewed a series of 24 patients who presented at an average time of 39 months with established fractures or nonunions of the olecranon from various causes including trauma, prior olecranon osteotomy, and pathologic fractures who were converted to TEA or had prior TEA [6]. These patients were treated with excision and triceps advancement, tension band with or without bone graft, or suture fixation. One patient in their series had failure of their tension band construct and underwent revision with a non-bridging dorsal plate at the level of the ulnar component and bone grafting. There have been no reports to our knowledge on immediate conversion to TEA following olecranon osteotomy in attempted ORIF of intraarticular distal humerus fractures.

We present a case of a 60 -year-old female acutely converted to TEA following attempted ORIF of a comminuted displaced intra-articular distal humerus fracture (Figure 1) utilizing an olecranon osteotomy. Periarticular plate and screw fixation bridging the ulnar component of the TEA and its cement mantle was used to repair the olecranon osteotomy site, restoring the extensor mechanism.

The patient had medical history of acromegaly, transphenoidal pituitary resection, and was compliant with 
chronic steroid replacement. She presented six days after falling off her porch for evaluation of left elbow and wrist pain. Clinical and radiographic examination demonstrated a comminuted intra-articular distal humeral fracture as well as a three-part intra-articular distal radius fracture. She described herself as a homemaker. After a preoperative discussion regarding both TEA and ORIF, specifically discussing lifting restrictions present with TEA, the patient preferred ORIF to TEA.

\section{Indications/Contraindications}

Open reduction internal fixation of displaced distal humeral fractures is contraindicated in patients who are not medically cleared for surgery, and in patients who cannot comply with postoperative protocols (i.e. advanced dementia).

TEA is indicated in the setting of distal humeral fractures in elderly patients (age > 65) when there is a displaced, comminuted intra-articular fracture that cannot be stabilized with internal fixation $[2,5,7,8]$. Amjid et al. reported on an unpublished survey of elbow surgeons around the world who identified their indications for intra-articular in the setting of acute distal humeral fractures. These included, non-rheumatoid patients over the age of 75 , patients with rheumatoid elbow disease at any age, patients with reduced life expectancy any age, patients with pathological bone at any age, and patients with degenerative elbow disease over the age of 60 [9].

\section{Technique}

The patient was placed supine with a bump under the ipsilateral scapula. A posterior surgical approach was made, initially exposing and isolating the ulnar nerve. Next, open reduction internal fixation of the distal humerus was attempted; with creation of para-tricipital windows and a chevron type olecranon osteotomy. Direct fracture visualization revealed multiple small fracture fragments not amenable to internal fixation. (Figure 2) Intra-operative decision was made to proceed with a semiconstrained TEA.

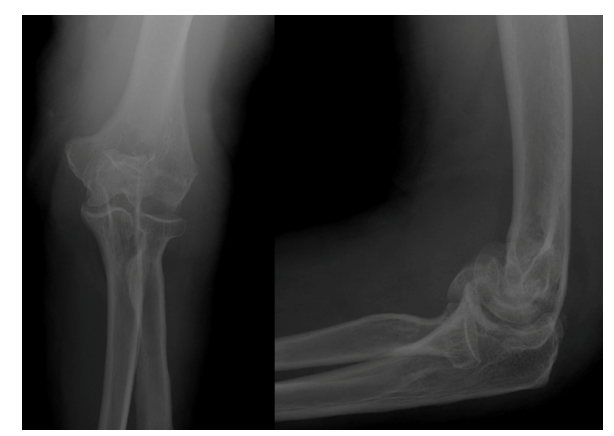

Figure 1. Radiographs demonstrate a displaced intra-articular distal humerus fracture.
In order to restore the extensor mechanism, the olecranon osteotomy site was reduced. The planned ulnar component was templated up against the ulna and a periarticular plate long enough to bypass the length of the TEA ulnar component and cement mantle was selected. (Figures 3 and 4) Compression plating of the olecranon osteotomy site was then performed. Following stable internal fixation of the olecranon osteotomy site, uneventful TEA followed. The distal radius was stabilized with internal bridge plating from the second metacarpal to the radial shaft, and this hardware was removed approximately three months postoperatively.

\section{Rehabilitation}

The patient was splinted for 2 weeks with the elbow at $\sim 60$ degrees of flexion and the forearm in neutral. Twoweeks postoperatively the splint was removed and the patient was allowed full active, active assist, and gentle passive range of motion. Supervised therapy was recommended.

\section{Peals and Pitfalls}

If the unfortunate need arises to reconstruct an olecranon osteotomy for conversion to TEA, stable internal fixation and compression across the osteotomy site will lead to improved osteotomy healing rates. Spanning the ulnar component allows the surgeon to avoid violating the cement mantle, purposefully or accidently, which could occur when attempting a tension band construct or plate and screw construct at the site of the ulnar implant.

The decision to proceed with acute TEA in the setting of distal humeral fractures should ideally be made preoperatively to eliminate the need to reconstruct the extensor mechanism.

\section{Outcomes \& Complications}

There were no intra-operative complications or complications at the 14-month postoperative time point. Clinically and radiographically the olecranon osteotomy site demonstrated union by 7 weeks. (Figure 5) The patient reported no elbow pain. Elbow ROM was fair; active flexion to 95 degrees, extension of (-)20 degrees, supination to 40 degrees and pronation to 75 degrees. (Figure 6).

\section{Conclusion}

The absolute number of distal humeral fractures performed in the elderly is increasing and surgeons should be prepared for unexpected situations when they arise. Optimally, the decision to proceed with TEA should be made preoperatively and olecranon osteotomy should be strictly avoided. Unfortunately, this cannot always relia- 


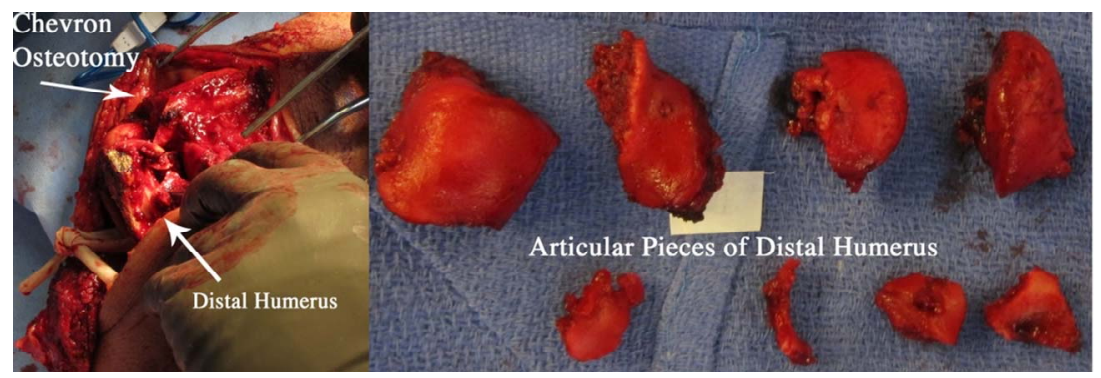

Figure 2. Distal humerus fracture fragments visualized following olecranon osteotomy.

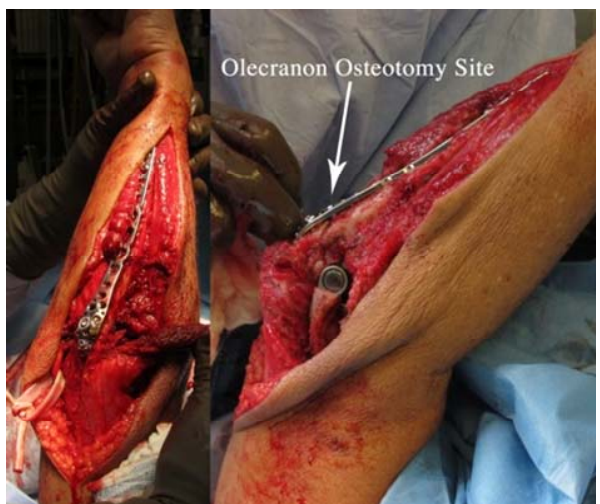

Figure 3. TEA and olecranon osteotomy repair using bridge plate.

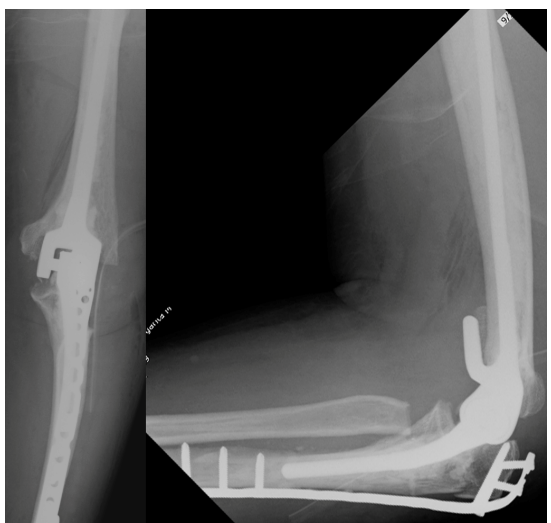

Figure 4. Intra-operative radiographs following bridging internal fixation and total elbow arthroplasty.

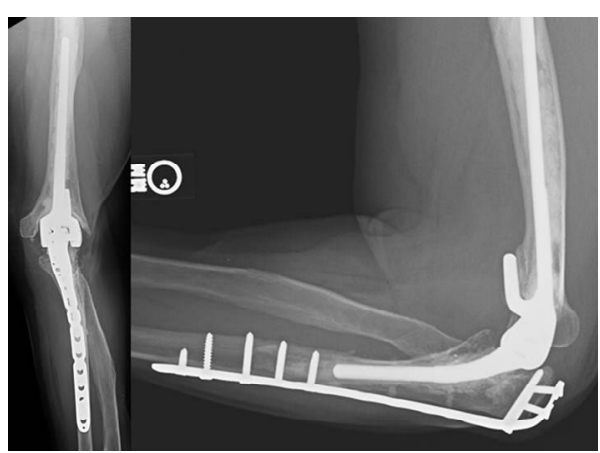

Figure 5. 14-month follow-up radiographs demonstrating olecranon osteotomy site union.

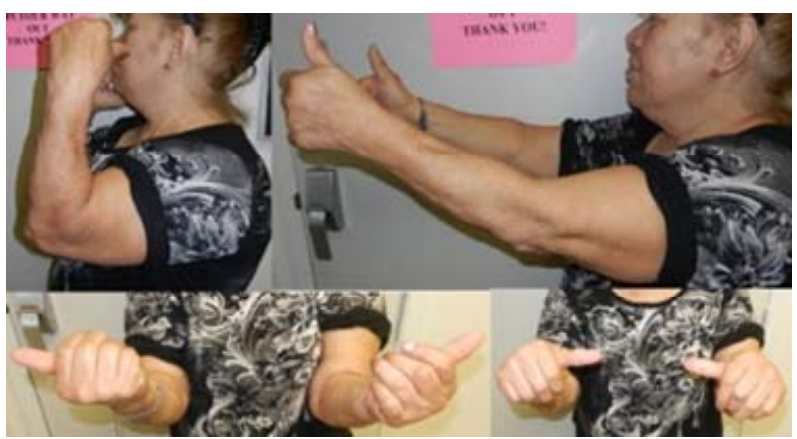

Figure 6. 14-month follow-up clinical photos demonstrating final range of motion.

bly be predetermined, and cases may arise following an olecranon osteotomy when the distal humeral fracture may not be reconstructible. This case presents a method to address the olecranon osteotomy (bypassing the ulnar component and its cement mantle) and perform acute TEA.

\section{REFERENCES}

[1] J. B. Jupiter, U. Neff, P. Holzach and M. Allgöwer, "Intercondylar Fractures of the Humerus. An Operative Approach," The Journal of Bone and Joint Surgery American Volume, Vol. 67, No. 2, 1985, pp. 226-239.

[2] A. Nauth, M. D. McKee, B. Ristevski, J. Hall and E. H. Schemitsch, "Distal Humeral Fractures in Adults," The Journal of Bone and Joint Surgery American Volume, Vol. 93, No. 7, 2011, pp. 686-700. doi:10.2106/JBJS.J.00845

[3] M. Palvanen, P. Kannus, S. Niemi and J. Parkkari, "Secular Trends in Distal Humeral Fractures of Elderly Women: Nationwide Statistics in Finland between 1970 and 2007," Bone, Vol. 46, No. 5, 2010, pp. 1355-1358. doi:10.1016/j.bone.2009.11.025

[4] M. D. McKee, C. J. H. Veillette, J. A. Hall, E. H. Schemitsch, L. M. Wild, R. McCormack, et al., "A Multicenter, Prospective, Randomized, Controlled Trial of Open Reduction-Internal Fixation versus Total Elbow Arthroplasty for Displaced Intra-Articular Distal Humeral Fractures in Elderly Patients," Journal of Shoulder and Elbow Surgery/American Shoulder and Elbow Surgeons, Vol. 18 No. 1, 2009, pp. 3-12.

[5] M. A. Frankle, D. Herscovici, T. G. DiPasquale, M. B. Vasey and R. W. Sanders, "A Comparison of Open Reduction 
and Internal Fixation and Primary Total Elbow Arthroplasty in the Treatment of Intraarticular Distal Humerus Fractures in Women Older than Age 65," Journal of Orthopaedic Trauma, Vol. 17, No. 7, 2003, pp. 473-480. doi:10.1097/00005131-200308000-00001

[6] G. Marra, B. F. Morrey, S. H. Gallay, M. D. McKee and S. O'Driscoll, "Fracture and Nonunion of the Olecranon in Total Elbow Arthroplasty," Journal of Shoulder and Elbow Surgery/American Shoulder and Elbow Surgeons, Vol. 15, No. 4, 2006, pp. 486-494.

[7] T. K. Cobb and B. F. Morrey, "Total Elbow Arthroplasty as Primary Treatment for Distal Humeral Fractures in Elderly Patients," The Journal of Bone and Joint Surgery.
American Volume, Vol. 79, No. 6, 1997, pp. 826-832.

[8] R. Gambirasio, N. Riand, R. Stern and P. Hoffmeyer, "Total Elbow Replacement for Complex Fractures of the Distal Humerus. An Option for the Elderly Patient," The Journal of Bone and Joint Surgery British Volume, Vol. 83, No. 7, 2001, pp. 974-978. doi:10.1302/0301-620X.83B7.11867

[9] A. Ali, S. Shahane and D. Stanley, "Total Elbow Arthroplasty for Distal Humeral Fractures: Indications, Surgical Approach, Technical Tips, and Outcome," Journal of Shoulder and Elbow Surgery/American Shoulder and Elbow Surgeons, Vol. 19, No. 2, 2010, pp. 53-58. 